\title{
High Gain Compact Hexagonal Fractal Antenna
}

\author{
Sneha Suresh Kadam ${ }^{l}$, Prof. Mahesh. S. Mathpati ${ }^{2}$ \\ ${ }^{I}$ ME-Student, Electronics \& Telecommunication Department, SVERI's College of Engineering, Pandharpur, \\ Maharashtra, India \\ ${ }^{2}$ Asst.Prof, Electronics \& Telecommunication Department, SVERI's College of Engineering, Pandharpur, \\ Maharashtra, India
}

\begin{abstract}
This paper proposes the concept of compact hexagonal fractal multi-band antenna. By using the compact hexagonal fractal antenna it is possible to achieve the directional pattern with high gain. The coaxial feeding technique is used for designing the antenna. This hexagonal antenna is used for different wireless application. The working frequency band covers a large number of wireless communication applications including GPS (1.6GHz), Bluetooth $(2.4 \mathrm{GHz}) \& W L A N(3.6 \mathrm{GHz})$. With hexagonal structure it is possible to configure the multi-band frequency with high gain. Antenna design has been designed and analyzed by using the software Ansoft's HFSS. Comparative results of simulated parameters like gain, return loss, directivity, VSWR, which had presented in this paper.
\end{abstract}

Keywords: Directivity, Fractal antenna, gain, HFSS, microstrip, multi band Frequency.

\section{Introduction}

In view of the progress of the recent wireless communication systems and its application, wider bandwidth, multiband and low profile antennas are in great demand for both commercial and military applications. The fast increase of wireless communications leads to a huge demand in designing of a multiband antenna. Conventionally, each antenna operates at single or dual frequency bands, where different antenna is used for different applications. There are different configurations used for multiband antenna [3]. The fractal antenna geometry concept is a special technique used to design multiband antenna. The name "fractal", from the Latin 'fractus" meaning broken, was given to highly irregular sets by Benoit Mandelbrot in his foundational essay in 1975[1].Fractal is recursively generated structure having self-similar shape, which means that some of the part have same shape as the whole object but at the different scale. Due to the self-similarity property of the fractals they are especially suitable for the design of multiband frequency antenna. Due to the concept selfsimilarity and infinite complexities, the proposed geometry of the antenna is very versatile is in terms of polarization, radiation pattern, gain and bandwidth. In this paper the self-similarity property of hexagonal is used to achieve the multiband operation.

\section{Hexagonal Fractal Antenna}

The structure of fractal is important because effective length of fractal antenna can be increased while keeping the total area same. The hexagonal fractal antenna is implemented in the corner fed configuration with overlapping vertices to preserve the electrical self-similarity paramount in multi band design [3]. There are many features of fractal geometries like, infinite complexity, fractional dimension and self-similarity. These characteristic features of fractals can be utilized in antenna design to get the following advantages:

Better efficiency: Fractals have sharp corners and edges that cause quick changes in the direction of current and hence improve radiation. Therefore fractals are efficient radiators of electromagnetic energy [2].

Multiband antennas: Due to the self-similarity property of fractals there are multiple arrangement of the geometry in a fractal object and hence they can be utilized for multiband antennas [2].

Size: Compact size compared to antennas of expected designs, while maintaining good to exceptional efficiencies and gains.

\subsection{Design of hexagonal patch antenna}

The Hexagonal microstrip antenna is designed at $2.4 \mathrm{GHz}$ frequency. The three essential parameters for the design of a Hexagonal patch Antenna are, The resonant frequency (fo) of the antenna must be selected suitably depending on its application. The resonant frequency selected for design is $2.4 \mathrm{GHz}$. Dielectric constant of the substrate ( $(\varepsilon)$ is selected for design of an antenna is glass epoxy which has a dielectric constant of 4.4. Height of dielectric substrate (h): For the antenna to be used in cellular phones, it is essential that the antenna is not bulky. Hence, the height of the dielectric substrate is selected as $1.6 \mathrm{~mm}$. Side length (a) of Hexagonal antenna can be calculated as, $\mathrm{a}=6 \mathrm{fr} / 2 \varepsilon^{1 / 2}$ 
Where $(\varepsilon r)$ is the dielectric constant.

fr is the resonant frequency.

$\mathrm{Fr}=2.4 \mathrm{GHz}$

$\varepsilon r=4.4$

$\mathrm{h}=1.6 \mathrm{~mm}$

The feed point can be calculated as,

Feed point=Length of patch/4.

The optimizations are done to achieve the best results. The first four iteration of hexagonal fractal antenna is shown in below figure which shows that the area remains same but length of antenna get increases due to iterations.

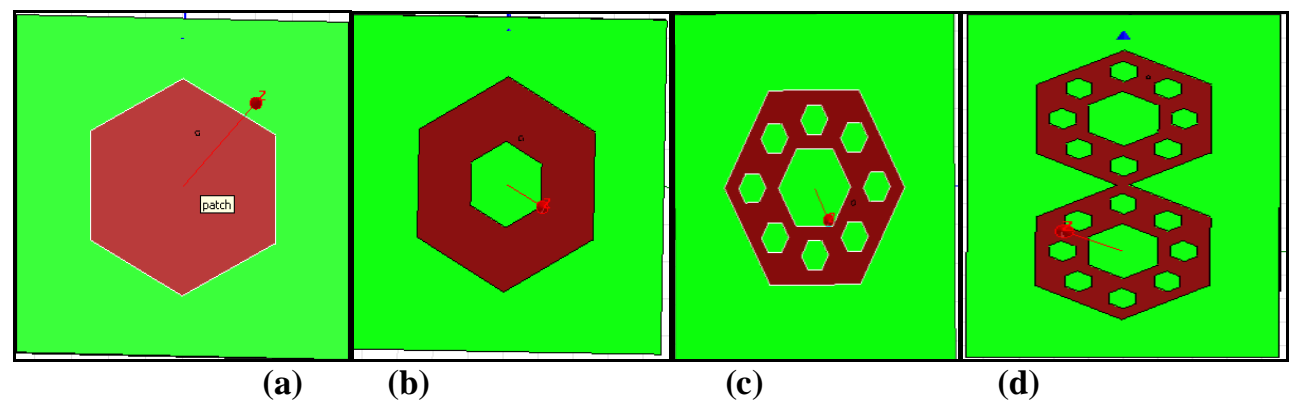

Fig -1: (a), (b), (c) and (d) First Four iterations of the hexagonal fractal Antenna

III. Simulated Results

The antenna is designed at $2.4 \mathrm{GHz}$ frequency. The first three iterations of the corner-fed hexagonal fractal dipole antenna are simulated by using HFSS software and results are shown below.

\subsection{Return Loss}

The Return Loss for the $2^{\text {nd }}$ iteration and $3^{\text {rd }}$ iteration of the hexagonal fractal antenna are plotted in Figure2, (a) and (b) respectively. The Hexagonal fractal Antenna produces a low return loss compared to the Sierpinski Carpet Fractal Antenna

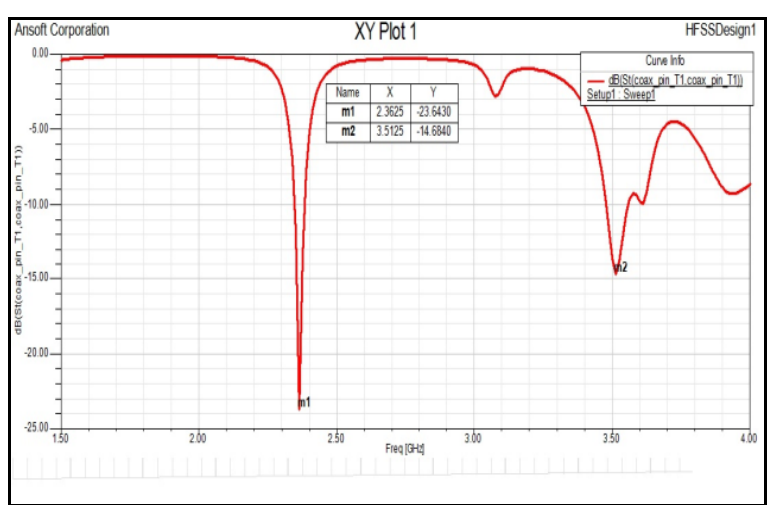

(a)

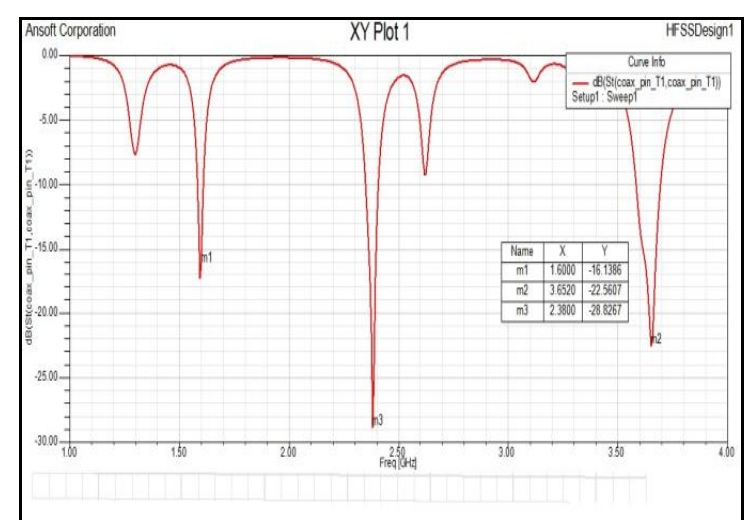

(b)

Fig- 2: (a), (b) Return Loss of $2^{\text {nd }}$ and $3^{\text {rd }}$ Iteration for Hexagonal Fractal Antenna

\subsection{VSWR}

Voltage Standing Wave Ratio (VSWR) is ratio between maximum voltage and minimum voltage along transmission line. It is derived from incident and reflected wave level. [8] VSWR increases if there is mismatch between the antenna and transmission line. If there are good matching then it is minimum. The VSWR is given by:

VSWR $=Z_{0}\left(1+S_{11}\right) /\left(1-S_{11}\right)$ 


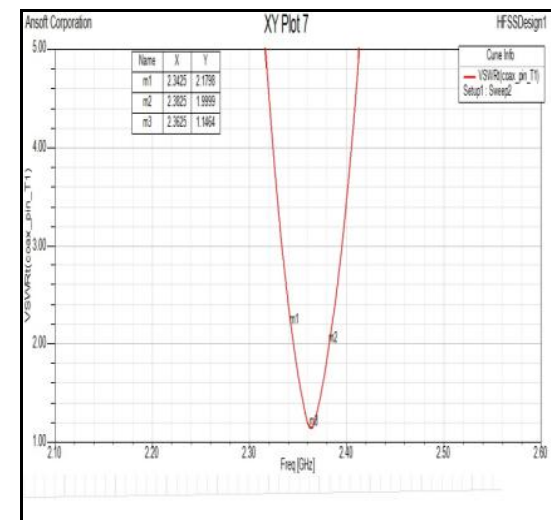

(a)

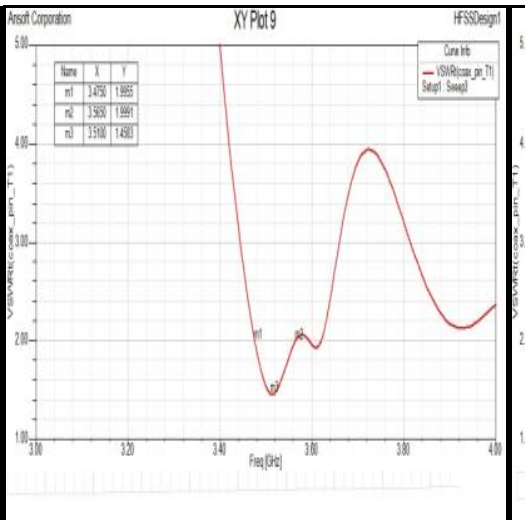

(b)

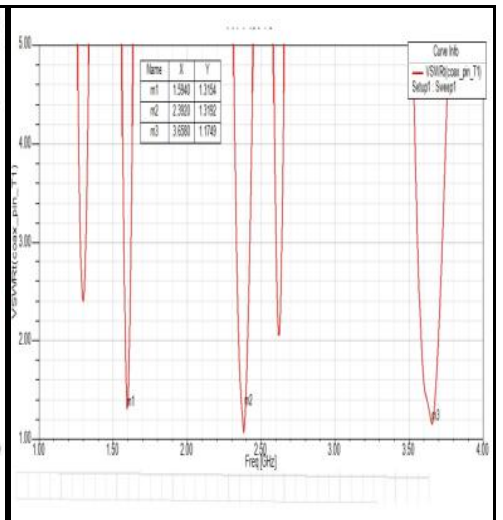

(c)

Fig-3 (a) VSWR for $2^{\text {nd }}$ Iteration of Hexagonal Fractal Antenna at $2.4 \mathrm{GHz}$, (b) VSWR for $2^{\text {nd }}$ Iteration of Hexagonal Fractal Antenna at 3.6GHz, (c) VSWR for $3^{\text {rd }}$ Iteration of Hexagonal Fractal Antenna

\subsection{Directivity}

Directivity, D is an significant parameter that shows the ability of the antenna focusing radiated energy. Directivity is a ratio of maximum radiated to radiate by reference antenna. Reference antenna is an isotropic radiator where the radiated energy is same in all the direction and have directivity of 1.Directivity can be defined as,

$\mathrm{D}=\mathrm{Fmax} / \mathrm{Fo}$

Where,

Fmax= Maximum radiated energy

$\mathrm{Fo}=$ Isotropic radiator radiate energy

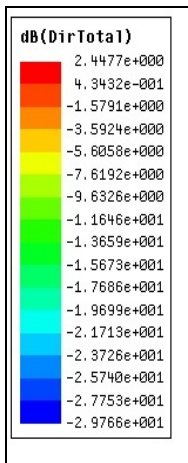
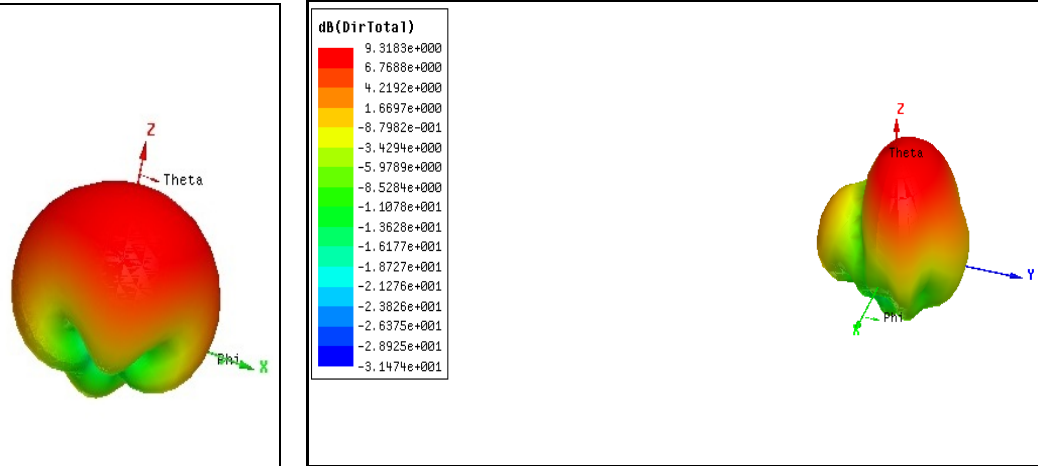

Fig-4:(a) and (b) Directivity for $2^{\text {nd }}$ and $3^{\text {rd }}$ Iteration of Hexagonal Fractal Antenna

The Hexagonal fractal antenna is designed for the four iterations i.e. $0^{\text {th }}$ iteration, $1^{\text {st }}$ iteration, and $2^{\text {nd }}$ iteration and $3^{\text {rd }}$ iteration. And the results are noted for the different parameters as shown below,

Table -1: Iteration wise result of compact hexagonal fractal antenna.

\begin{tabular}{|l|l|l|l|l|l|}
\hline Sr.No & $\begin{array}{l}\text { Iteration } \\
\text { wise results }\end{array}$ & $\begin{array}{l}\text { Frequency } \\
(\mathrm{GH})\end{array}$ & Return Loss & VSWR & $\begin{array}{l}\text { Gain } \\
(\mathrm{dB})\end{array}$ \\
\hline 1$)$ & $0^{\text {th }}$ iteration & 2.52 & -22.13 & 1.16 & 3.84 \\
\hline 2$)$ & $1^{\text {st }}$ iteration & 2.45 & -17.81 & 1.26 & 3.28 \\
\hline \multirow{2}{*}{$3)$} & \multirow{2}{*}{$2^{\text {nd }}$ iteration } & 2.36 & -23.64 & 1.14 & \multirow{2}{*}{2.45} \\
\cline { 3 - 5 } & \multirow{3}{*}{$4)$} & 3.51 & -14.68 & 1.45 & \multirow{2}{*}{9.318} \\
\cline { 3 - 5 } & \multirow{2}{*}{$3^{\text {rd }}$ iteration } & 1.6 & -16.138 & 1.315 & \\
\cline { 3 - 5 } & & 2.38 & -28.826 & 1.319 & 1.174 \\
\cline { 3 - 5 } & & 3.65 & -22.506 & & \\
\end{tabular}

The above table shows that as iteration increases the fundamental frequency is shifted to the lower side and which is used for multiband application. The VSWR is minimum for $3^{\text {rd }}$ iteration as compared to first three iterations. The high gain is achieved at $3^{\text {rd }}$ iteration. 


\section{Conclusion}

In this paper compact hexagonal fractal antenna has designed and results are simulated by using HFSS software are shown .Which possess multiband behavior similar to the sierpinki gasket antenna. This compact hexagonal fractal antenna covers the frequency range from $1.6 \mathrm{GHz}$ to $3.6 \mathrm{GHz}$. With this arrangement it is possible to modernize radiation pattern according to the requirement. However, an optimum selection of position of the feed is important for better results. The gain is increased by increasing the iteration and keeping its physical length same. Simulated results have shown a high gain, low return loss as iterations are increases. Also it shows good results compared with the simple patch antenna. The more frequency range with high gain can be achieved by increasing array elements.

\section{References}

[1]. B. B. Mandelbrot, "The Fractal Geometry of Nature", New York, W. H. Freeman, 1983

[2]. Muhammad Waqas1, Zubair Ahmed2, Mojeeb Bin Ihsan "Multiband Sierpinski Fractal Antenna" 2009 IEEE

[3]. N.A. Saidatul, A.A.H. Azremi, P.J Soh, “A Hexagonal Fractal Antenna for Multiband Application" International Conference on Intelligent and Advanced Systems 2007.

[4]. P. W. Tang and P. F. Wahid, "Hexagonal Fractal Multiband Antenna" IEEE ANTENNAS AND WIRELESS PROPAGATION LETTERS,VOL.3, 2004.

[5]. Douglas H. Werner and SumanGangul, “An Overview' of Fractal Antenna Engineering Research”. IEEE Antennas and Propagation Magazine. Vol. 45, NO.I , February 2003.

[6]. Rahul Batra, P. L. Zade, DipikaSagne, "Design and Implementation of Sierpinski Carpet Fractal Antenna for Wireless Communication". International Journal of Scientific Research Engineering \&Technology (IJSRET) Volume 1 Issue3 pp 043-047 July 2012.

[7]. Rakesh Kumar,Gunjan Gupta "Study of Fractal Circular Patch Micro-Strip Antenna over Traditional Antenna" . International Journal of Innovative Technology and Exploring Engineering (IJITEE) ISSN: 2278-3075, Volume-1, Issue-5, October 2012.

[8]. N. A. Saidatul, A. A. H. Azremi, R. B. Ahmad, P. J. Soh and F. Malek, "MULTIBAND FRACTAL PLANAR INVERTED F ANTENNA (F-PIFA) FOR MOBILE PHONE APPLICATION". Progress In Electromagnetic Research B, Vol. 14, 127-148, 2009. 